\title{
Magnetic fields and transport processes
}

\author{
Patrick Eggenberger
}

\author{
Observatoire de Genève, Université de Genève, 51 Ch. des Maillettes, \\ CH-1290 Sauverny, Switzerland
}

\begin{abstract}
In this presentation, we briefly discuss how magnetic angular momentum transport in stellar radiative zones can be constrained by asteroseismic measurements. We focus on the internal rotational properties deduced from rotational splittings of solar-like oscillations and how they can be used to characterize angular momentum transport in stellar interiors.
\end{abstract}

Keywords: magnetic fields - transport processes - rotation

\section{Rotation of the solar interior}

\subsection{Helioseismic constraints}

Thanks to the study of solar oscillations, fundamental information can be obtained about the internal properties of the Sun. In particular, measurements of the rotational splittings of solar pressure modes enable to probe the rotation in the interior of the Sun down to about 0.2 solar radii (see e.g. Kosovichev 1988; Brown et al. 1989; Elsworth et al. 1995; Kosovichev et al. 1997; Couvidat et al. 2003; García et al. 2007). These key observational constraints for rotating stellar models aiming at taking into account the internal transport of angular momentum by various hydrodynamic and magnetohydrodynamic processes reveal two important features. First, in the convective envelope of the Sun, some differential rotation is observed as a function of the latitude together with a low degree of differential rotation as a function of the radius. Second, an almost uniform rotation is observed in the solar radiative zone. Interestingly, the transition from uniform rotation in the radiative zone to latitudinal differential rotation in the convective envelope takes place in a thin layer.

In addition to the constraints on the internal solar rotation deduced from pressure modes, the detection and characterization of gravity modes would offer a unique opportunity to deduce the rotation rate in the core of the Sun. Detecting solar gravity modes is of course a very difficult and challenging task, but the possibility to achieve such a detection becomes more actual with the increasing total duration of GOLF observations (e.g. Belkacem et al. 2009; Appourchaux et al. 2010; Belkacem 2011). This has been recently illustrated by a reported possible detection of gravity modes by Fossat et al. (2017). While the robustness of this detection is clearly not guaranteed (see e.g. Schunker et al. 2018; Appourchaux \& Corbard 2019), it is interesting to note that it suggests that the solar core rotates more rapidly than the more external part of the radiative zone. This directly raises the intriguing question of the possibility to find a transport mechanism able to simultaneously reproduce a fast rotating solar core and the nearly uniform rotation of the more external part of the solar radiative zone. 


\subsection{Angular momentum transport by hydrodynamic processes in the solar ra- diative zone}

To progress in the modelling of angular momentum transport in stellar interiors, one relies on the comparison between predictions of rotating stellar models and direct measurements of internal rotation rates. In this context, the rotation of the solar radiative zone as described above constitutes a fundamental observational constraint, which should be correctly reproduced by rotating stellar models. Concerning the modelling of rotational effects, the transport of angular momentum and chemical elements due to various hydrodynamic instabilities can be taken into account quite easily in a onedimensional stellar evolution code thanks to the assumption of shellular rotation (Zahn 1992). This simplifying assumption is based on the fact that the turbulence induced by rotation is much stronger in the horizontal than in the vertical direction in stellar radiative zones (Zahn 1992). This has for a direct consequence that the angular velocity remains approximately constant on an isobar, so that rotational effects can be studied quite easily within this framework using one-dimensional stellar evolution codes. In order to follow the evolution of a rotating stellar model, an additional equation is then solved for the evolution of the internal rotation profile simultaneously to the classical equations for the stellar structure. Moreover, the related transport of chemical elements is taken into account.

For the Sun, models computed within the framework of shellular rotation that take into account only the transport of angular momentum due to hydrodynamic processes predict a high degree of radial differential rotation in the radiative zone. The rapid increase of the angular velocity when the radius decreases in the solar radiative zone for such a rotating model is illustrated by the orange line in Fig. 1. This results from the combined action of the slow contraction of the central layers and the braking of the convective envelope by magnetized winds during the evolution on the main sequence. The transport of angular momentum in the radiative zone due to purely hydrodynamic processes (in particular the transport by the meridional circulation and the shear instability) is thus found to be insufficient in order to ensure the nearly uniform rotation deduced from rotational splittings of pressure modes. This is a clear indication that an additional efficient mechanism for the transport of angular momentum (in addition to meridional circulation and hydrodynamic instabilities) is required in radiative zones in order to correctly reproduce the helioseismic constraints on the internal rotation of the Sun.

\subsection{Magnetic angular momentum transport in the solar radiative zone}

Magnetic fields offer an interesting possibility to efficiently transport angular momentum in stellar radiative zones without leading to an efficient transport of chemical elements. Such an efficient transport of chemicals is indeed disfavored by the moderate depletion of light elements observed at the surface of the Sun. A weak magnetic field can be responsible of significant changes in the internal rotation of stars (e.g. Mestel 1953). In particular, large-scale magnetic fields of fossil origin are able to ensure an efficient coupling in the solar interior that is needed to explain the nearly uniform rotation of its radiative zone (e.g. Mestel \& Weiss 1987; Charbonneau \& MacGregor 1993; Rüdiger \& Kitchatinov 1996; Spada et al. 2010). While such large-scale fields can explain the absence of differential rotation in the radial direction in the radiative zone of the Sun, they also lead to the question of the coupling they induce between the rotational properties of the solar radiative zone and the convective envelope. This raises the key issue of the helioseismic constraint of a thin tachocline for the transition from latitudinal differential rotation in the convective envelope to uniform rotation in the radiative interior.

One possibility to reproduce this sharp transition in the rotation profile is to invoked a large-scale magnetic field that is confined in the solar radiative interior. A theoretical model that explains how 


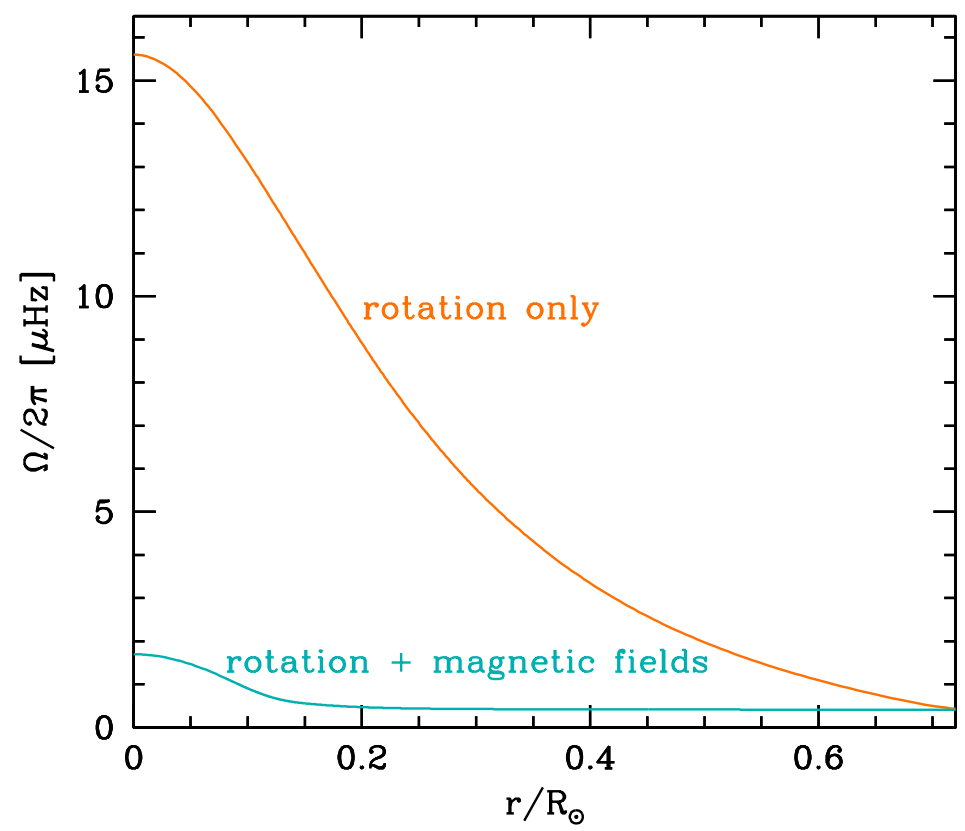

Figure 1: Rotation profiles in the solar radiative zone for two different rotating solar models. The orange line corresponds to a model that accounts for angular momentum transport by hydrodynamic mechanisms only. The green line indicates a solar model computed with both hydrodynamic angular momentum transport processes and magnetic fields in the framework of the Tayler-Spruit dynamo (Spruit 2002). Adapted from Eggenberger et al. (2005, 2019a)

such a confinement of the field could be obtained has been proposed by Gough \& McIntyre (1998). To test this scenario, numerical simulations have been computed leading to different results. Some simulations indicate that such a confinement is not easy to realize: due to magnetic diffusion, the initially confined magnetic field is able to connect to the convective envelope, resulting then in a propagation of differential rotation in the radiative interior, which is in contradiction with helioseismic measurements (see e.g. Brun \& Zahn 2006; Strugarek et al. 2011). However, other numerical simulations indicate that the configuration proposed by Gough \& McIntyre (1998) could be achieved (see e.g. Acevedo-Arreguin et al. 2013; Wood \& Brummell 2018).

Even if the large-scale magnetic field can be confined to the radiative zone, it is interesting to note that a certain degree of coupling is needed between the solar radiative interior and the convective envelope. Indeed, a braking of the convective envelope by magnetized winds was at work during the past evolution of the Sun. Without such a coupling between the radiative and convective zones, a lower rotation rate would be observed in the convective envelope than in the radiative interior of the Sun. An increase of the viscosity in the solar radiative zone seems also to be needed in addition to a large-scale fossil field to reproduce quantitatively the low degree of radial differential rotation deduced from rotational splittings of pressure modes (Rüdiger \& Kitchatinov 1996; Spada et al. 2010). In this context, magnetic instabilities can play an important role for the transport of angular momentum in radiative zones.

Various magnetic instabilities can take place in stellar interiors. In stellar radiative zones, the Tayler instability (Tayler 1973) seems to be the first instability to set in (Spruit 1999). A small-scale time-dependent dynamo mechanism based on this Tayler instability of toroidal fields was proposed by Spruit (2002). An important feature of this mechanism (refered to as the Tayler-Spruit dynamo) is that the amplitudes of the associated magnetic fields are much larger horizontally than vertically. The transition from uniform rotation in the radiative interior to differential rotation as a function of 
the latitude in the convective zone of the Sun can then be correctly accounted for by this magnetic transport process. The possibility for this kind of small-scale dynamo to operate in stellar radiative zones has been tested with the computation of numerical simulations. Different results have then been obtained: simulations performed by Braithwaite (2006) indicate that this process can operate in stellar radiative interiors, while simulations performed by Zahn et al. (2007) indicate that this dynamo mechanism is not present in stellar interiors. These different conclusions mainly reflect differences in the input parameters used in the simulations, in particular regarding magnetic diffusivity. The challenge from the point of view of numerical simulations is to perform computations under more realistic stellar conditions to have a better idea of the possibiliy for such a small-scale dynamo to be at work in stellar radiative zones (see e.g. Braithwaite \& Spruit 2017).

In addition to numerical simulations, angular momentum transport processes in stellar interiors can be tested by confronting their predicted rotation profiles to internal rotation rates deduced from helio and asteroseismic measurements. In particular, the helioseismic constraints on the internal rotation of the Sun briefly recalled above in Sect. 1.1 must be correctly reproduced by rotating solar models. In the specific case of the Tayler-Spruit dynamo, the efficient additional transport of angular momentum related to this process leads to a rotation profile in good agreement with the approximately uniform rotation in the solar radiative zone deduced from measurements of rotational splittings of pressure modes (Eggenberger et al. 2005, 2019a). This is the first important observational constraint that has to be reproduced by any candidate transport process aiming at describing angular momentum transport in radiative interiors of stars. The rotation profile of a solar model computed with both hydrodynamic transport processes and the Tayler-Spruit dynamo is shown by the green line in Fig. 1.

Pressure modes enable to probe the internal rotation of the Sun down to about 0.2 solar radii. The rotation rate in the more central layers could be revealed through the detection of gravity modes. As mentioned above, reported detections of gravity modes suggest that the solar core could rotate more rapidly than the part of the solar radiative zone probed by pressure modes (García et al. 2007, 2011; Fossat et al. 2017). While this result is clearly hypothetical due to the difficulty of detecting gravity modes (see e.g. Schunker et al. 2018; Appourchaux \& Corbard 2019), it is interesting to note that an increase in the angular velocity of the solar central layers can be reproduced simultaneously to the almost uniform rotation of the more external part of the radiative zone by models accounting for angular momentum transport by magnetic instabilities (Eggenberger et al. 2019a). This is illustrated in Fig. 1, with the green line exhibiting an increase in the angular velocity in the central layers (typically below about 0.15 solar radius). This result is a direct consequence of the strong inhibiting effect of the chemical gradients related to nuclear reactions in the central layers on the efficiency of angular momentum transport by magnetic instabilities. A faster rotating solar core is not expected in the case of a large-scale magnetic field able to explain the approximately uniform rotation of the more external part of the radiative interior revealed by pressure modes. This key difference underlines the importance of having a firm detection of solar gravity modes for the modelling of magnetic angular momentum transport in stellar interiors.

\section{Transport of angular momentum in the radiative interior of main-sequence stars}

\subsection{Internal rotation of solar-type stars}

As discussed above, helioseismic measurements show that an efficient mechanism for angular momentum transport in radiative zones is needed in addition to the transport of angular momentum by meridional currents and the shear instability. One can then wonder whether the Sun constitutes a pecu- 
liar case in this context or whether such an efficient process for internal angular momentum transport is also required for other stars. In particular, do asteroseismic measurements of solar-type stars on the main sequence also indicate that rotation is approximately uniform in their radiative interiors? In the case of main-sequence solar-type stars, it is not easy to constrain radial differential rotation from measurements of the rotational splittings of the detected pressure modes (see e.g. Lund et al. 2014; Schunker et al. 2016). However, by combining these asteroseismic data with an independent determination of the surface rotation velocity of the star, one can then deduce some constraints on the difference in rotation between the convective envelope and the radiative zone. The independent information on the surface rotation rate can be provided by variations in luminosity due to spots at the stellar surface or by spectroscopic measurements. While being dominated by rotation in the external part of the stellar interior, the mean rotation rate deduced from asteroseismic measurements of rotational splittings of pressure modes retains some sensitivity to the internal rotation of the star. In this context, the presence of radial differential rotation can be revealed through different values for the asteroseismic and surface rotation rates.

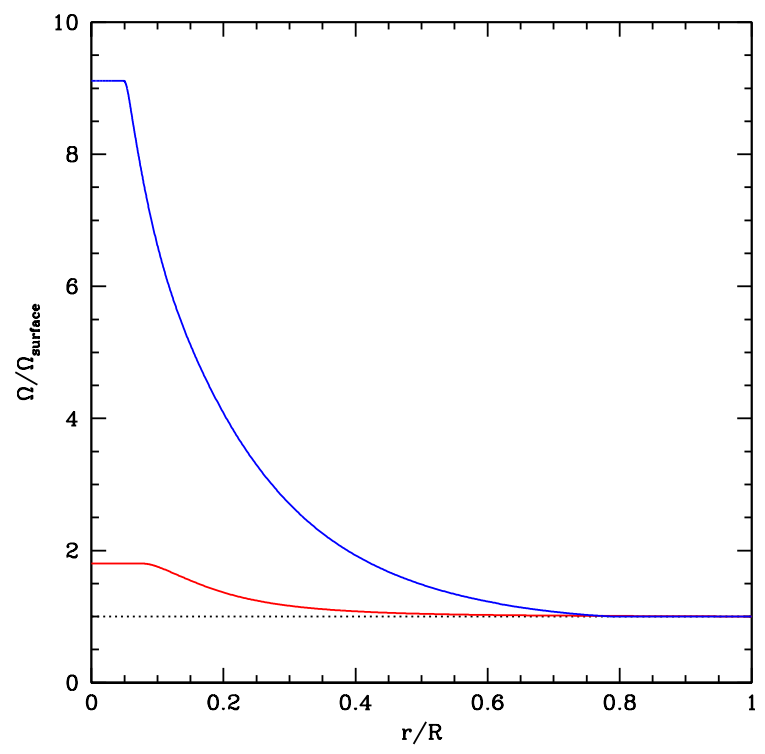

Figure 2: Angular rotation velocity normalized to its surface value in the interior of two different main-sequence rotating models accounting for angular momentum transport by meridional currents and the shear instability. The blue and red lines correspond to $1.2 \mathrm{M}_{\odot}$ and $1.5 \mathrm{M}_{\odot}$ models, respectively.

This kind of analysis has been performed for main-sequence solar-type stars (e.g. Benomar et al. 2015; Nielsen et al. 2015). Very small differences have then been obtained between the rotation rate deduced from asteroseismic data (this is done by assuming solid-body rotation in the radiative zone) and the surface rotation rate for the vast majority of the stars studied by Benomar et al. (2015) and Nielsen et al. (2015). Similarly to the solar case, these results indicate that the radiative interior of solar-type main-sequence stars rotates at a similar rate as the convective envelope. These studies thus suggest that an efficient internal angular momentum transport of is also present in these stars. Comparison with rotational properties predicted by stellar models that only account for hydrodynamic transport processes is then needed to determine whether an additional efficient angular momentum transport mechanism is also required for these stars as it is the case for the Sun.

The typical rotation profiles of two rotating models of main-sequence solar-type stars computed by only taking into account hydrodynamic transport processes are shown in Fig. 2. The blue line 
corresponds to a $1.2 \mathrm{M}_{\odot}$ model, while the red line indicates a more massive $1.5 \mathrm{M}_{\odot}$ model. Due to an efficent braking of the surface by magnetized winds, rotating models of main-sequence stars with deep convective envelopes develop a significant degree of radial differential rotation in the external part of the radiative zone when only hydrodynamic transport processes are taken into account (blue line in Fig. 2). For these stars, an efficient transport mechanism is thus needed in addition to the angular momentum transport by meridional circulation and the shear instability to correctly reproduce the asteroseismic constraints. Main-sequence stars with shallower convective envelopes (typically more massive stars) are less subject to such an efficient braking of their surface by magnetized winds. Consequently, a lower degree of radial differential rotation is predicted for these stars by models with purely hydrodynamic transport processes (red line in Fig. 2). Moreover, the gradient of angular velocity in these stars is mainly located close to the border with the convective core and not in the external part of the radiative zone. It will thus be difficult to detect this kind of radial differential rotation by comparing a mean asteroseismic rotation rate (which is dominated by rotation in the external layers) with an independent measurement of the surface rotation rate. For these more massive main-sequence solar-type stars, finding a similar value for the asteroseismic and surface rotation rates does not necessarily mean that an efficient transport process is needeed in addition to hydrodynamic transport mechanisms.

\subsection{Effects of an efficient process for the transport of angular momentum on the transport of chemical elements}

As discussed above, asteroseismic measurements suggest that an efficient additional angular momentum transport process is needed in the radiative zones of solar-type stars with deep convective envelopes. This has interesting consequences on chemical elements mixing in radiative interiors and hence on the main-sequence lifetimes of these stars. Indeed, an efficient transport of angular momentum in radiative zones results in a very low degree of radial differential rotation, which implies an inefficient transport of chemicals by the shear instabilitiy. Moreover, due to the strong braking of the surface by magnetized winds, the surface velocities of these stars rapidly decrease during the first part of their evolution on the main sequence. These low rotation rates then lead to a low efficiency of the transport of chemicals by meridional circulation. A global decrease of the efficiency of rotational mixing is then obtained in the interiors of solar-type stars when an efficient magnetic angular momentum transport is taken into account (Eggenberger et al. 2010).

This is illustrated in Fig. 3, which shows the evolution of the surface helium mass fraction as a function of the central hydrogen mass fraction for three different $1 \mathrm{M}_{\odot}$ models. The magenta line corresponds to a non-rotating model. In this case, a decrease of the surface helium abundance is observed during the main-sequence evolution due to atomic diffusion. The orange line corresponds to a model computed with the same input parameters except for the inclusion of the transport of angular momentum and chemicals by meridional currents and the shear instability. As shown in Fig. 3, rotational mixing is found to counteract the effects of atomic diffusion so that the decrease in the helium surface abundance is less pronounced for the rotating model computed with only hydrodynamic transport processes (orange line) than for the non-rotating model (magenta line). When angular momentum transport by magnetic instabilities is taken into account in addition to hydrodynamic transport mechanisms (green line), the values of the surface helium abundance are then found to be more similar to the ones of the non-rotating model. This is a direct consequence of the decrease in the efficiency of rotational mixing when the effects of magnetic fields are taken into account.

The effects of a magnetic angular momentum transport process are of course not restricted to the surface abundances of solar-type stars, since they also impact the main-sequence lifetimes of these stars. Indeed, a model of a solar-type star computed with atomic diffusion will exhibit a shorter 


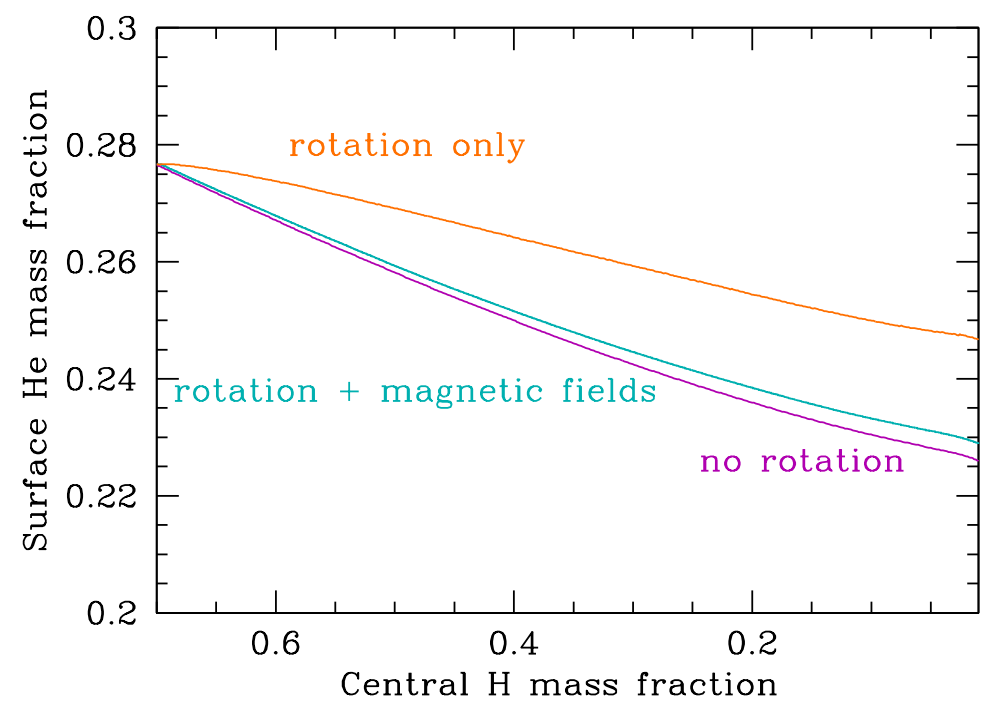

Figure 3: Surface helium abundance as a function of core hydrogen mass fraction during the mainsequence evolution. These $1 \mathrm{M}_{\odot}$ models are computed with a solar chemical composition and take into account atomic diffusion. The magenta line corresponds to a non-rotating model. The orange line indicates a model that takes into accout the internal transport of angular momentum due to hydrodynamic processes. In addition to hydrodynamic processes, the green line corresponds to a model computed with the transport of angular momentum by the Tayler instability in the framework of the Tayler-Spruit dynamo. Adapted from Eggenberger et al. (2010).

main-sequence lifetime than a model without atomic diffusion. Since rotational mixing couteracts the effects of atomic diffusion, including hydrodynamic transport processes results in an increase of the main-sequence lifetime of rotating models. By reducing the effects of rotational mixing, magnetic angular momentum transport then leads to main-sequence lifetimes that are more similar to the ones of non-rotating models.

The impact of the transport of angular momentum by magnetic fields can be quite different in more massive stars compare to the case of solar-type stars discussed above. While in both cases an efficient angular momentum transport results in a strong decrease of the mixing by the shear instability, the transport by meridional currents can be quite different. This is related to the fact that more massive stars are characterized by shallower convective envelope and thus do not experience a significant braking of their surface by magnetized winds. Consequently, they can exhibit high rotation velocities on the main sequence that lead to a strong chemical mixing by meridional circulation. For these stars, a flatter rotation profile leads to a more efficient chemical elements transport by meridional circulation. The latter increase in the transport efficiency of meridional currents can compensate for, and can even be more important than, the decrease of the transport by the shear instability. Models of massive stars computed with both hydrodynamic and magnetic transport processes can then be more efficiently mixed than models that only take into account hydrodynamic processes (e.g. Maeder \& Meynet 2005).

\section{Transport of angular momentum in subgiant stars}

In addition to purely hydrodynamic transport process, an efficient mechanism for angular momentum transport in radiative zones is required in order to reproduce the internal rotation profile of the Sun and the asteroseismic constraints available for solar-type stars. The next question is to determine 
whether such an additional transport process is also needed during the post-main sequence evolution of these stars. To answer this question, we first consider the transport of angular momentum in subgiant stars. For these evolved stars, key information can be obtained on their internal rotation thanks to the measurements of rotational splittings of mixed oscillation modes. Thanks to their sensitivity to both external and central layers, these mixed modes are of peculiar interest to determine the core and surface rotation rates of post-main sequence stars. This is exactly what is needed to determine the internal angular momentum transport during the post-main sequence evolution. In particular, asteroseismic measurements of both core and surface rotation rates have been obtained for six subgiant stars by Deheuvels et al. (2014). These measurements are then compared to predictions of rotating models to constrain the internal transport of angular momentum.

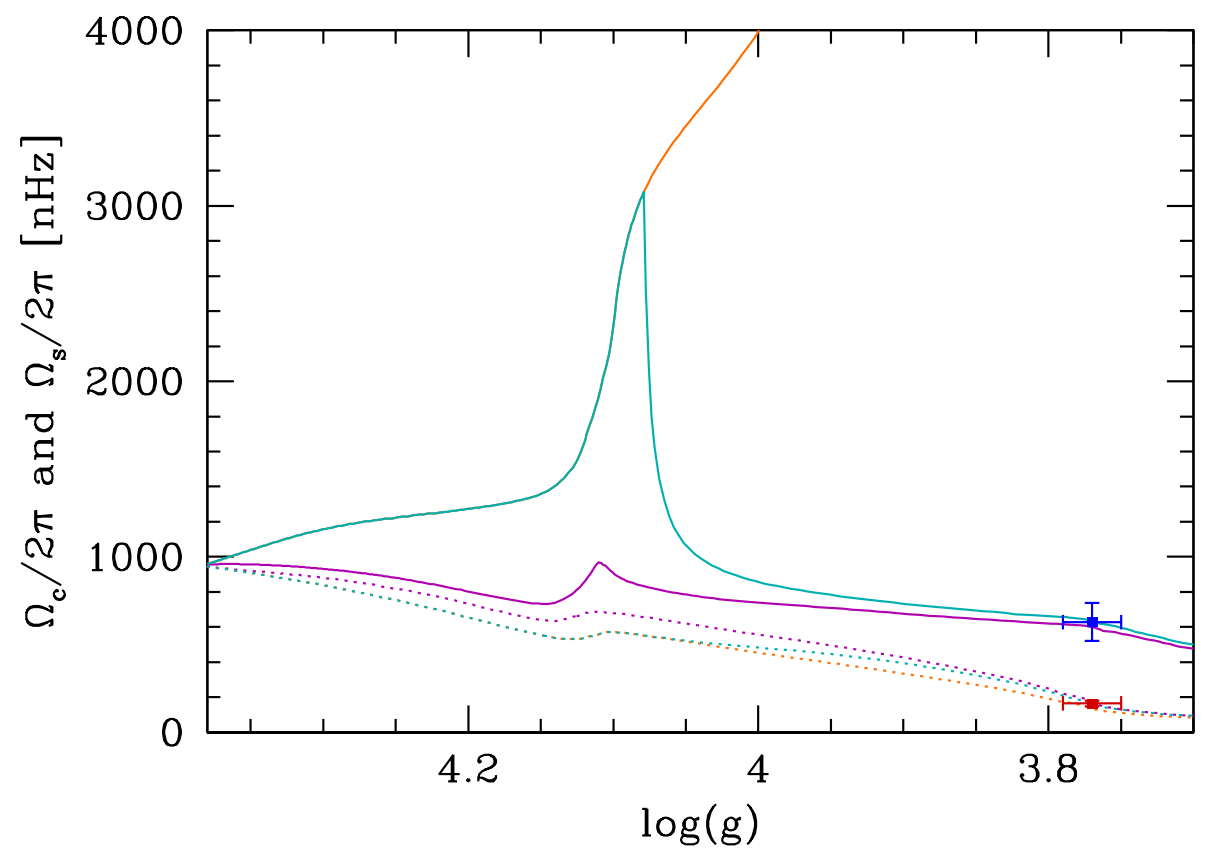

Figure 4: Core (continuous lines) and surface (dotted lines) rotation rates as a function of surface gravity for three different rotating models of the subgiant KIC 8702606. The orange line corresponds to a rotating model computed with angular momentum transport by meridional circulation and the shear instability. The magenta line corresponds to the same model except for the inclusion of an additional viscosity $\nu_{\text {add }}$ of $1.7 \times 10^{4} \mathrm{~cm}^{2} \mathrm{~s}^{-1}$ during the whole evolution of the star. The green line indicates a model computed with an additional viscosity $\nu_{\text {add }}$ of $1.7 \times 10^{4} \mathrm{~cm}^{2} \mathrm{~s}^{-1}$ but only during the post-main sequence evolution. The blue and red squares correspond to the core and surface rotation rates of the subgiant KIC 8702606 as determined by Deheuvels et al. (2014). Adapted from Eggenberger et al. (2019b).

From a comparison with models that only account for hydrodynamic transport processes, we first find that the transport associated to meridional circulation and the shear instability is not efficient enough to correctly reproduce the asteroseismic observations. This is illustrated by the orange lines in Fig. 4 for one of the subgiant studied by Deheuvels et al. (2014). Figure 4 indeed shows that a model with only hydrodynamic transport processes that correctly reproduces the observed surface rotation rate (orange dotted line) predicts a core rotation rate (continuous orange line) that is more than one order of magnitude larger than the observed one. This is due to the insufficient coupling ensured by meridional currents and the shear instability to prevent the core from reaching high values of rotation rates as a result of the post-main sequence contraction of the central layers. We conclude that, similarly to the case of the Sun and main-sequence solar-type stars, an additional efficient angular 
momentum transport process is also at work in the radiative zones of subgiants (Eggenberger et al. 2019b).

The next point that has to be investigated is how asteroseismic measurements of subgiants can be used to constrain the efficiency of the needed additional transport process during the post-main sequence evolution. This can be done by introducing a constant viscosity $\nu_{\text {add }}$ in the equation describing the internal transport of angular momentum in addition to the advective transport of angular momentum by meridional currents and the diffusive transport by the shear instability. Such a simple assumption of a constant additional viscosity is useful to investigate whether quantitative constraints on the efficiency of the post-main sequence angular momentum transport can be deduced from asteroseismic constraints on the internal rotation of subgiants. We have shown that the efficiency of angular momentum transport can be precisely determined for the subgiants studied by Deheuvels et al. (2014). The determination of the efficiency of this transport during the subgiant phase is indeed found to be insensitive to the rotational history of the star and in particular to the uncertainties related to the modelling of rotation during the main sequence (Eggenberger et al. 2019b). This is illustrated by the green and magenta lines in Fig. 4. These lines correspond to models computed with the same additional viscosity of $1.7 \times 10^{4} \mathrm{~cm}^{2} \mathrm{~s}^{-1}$ during the post-main sequence but different assumptions for the angular momentum transport on the main sequence. The green and magenta lines are indeed computed without and with an additional viscosity of $1.7 \times 10^{4} \mathrm{~cm}^{2} \mathrm{~s}^{-1}$ during the main sequence, respectively. As shown in Fig. 4, the rotational properties of younger subgiants (typically stars characterized by a $\log (g)$ close to 4 ) retains some sensitivity to their rotational history and could thus be used to characterize the transport of angular momentum on the main sequence.

Another important question that can be investigated with the simple assumption of an additional constant viscosity is the change in the mean efficiency of the transport of angular momentum with global parameters (in particular the stellar masses and ages). The angular momentum transport efficiency is then found to increase with the mass of the star during the subgiant phase. Concerning the change of the transport efficiency with the evolutionary stage, a decrease of the mean efficiency of the internal transport of angular momentum is found when the star evolves during the subgiant phase (Eggenberger et al. 2019b). These trends with the mass and the evolution of subgiant stars constitute important constraints that have to be correctly reproduced by any candidate for the additional angular momentum transport process.

In the context of the physical nature of the additional transport mechanism, we notice that, for two of the subgiants studied by Deheuvels et al. (2014), a rotation profile characterized by a sharp discontinuity seems to be in better agreement with asteroseismic data than a smooth rotation profile (see discussion in Sect. 6.4 of Deheuvels et al. 2014). The location of this sharp discontinuity is suggested to be close to the location of the hydrogen-burning shell. This could indicate that a decrease in the efficiency of the additional angular momentum transport process is associated to the presence of steep gradients of chemical composition. This is of prime interest for magnetic angular momentum transport. This seems indeed to favor transport by magnetic instabilities, which are sensitive to the inhibiting effects of chemical gradients (see Sect. 1.3), and to disfavor an efficient coupling ensured by large-scale magnetic fields. These are however very preliminary results that need to be confirmed by future asteroseismic observations of evolved stars.

\section{Transport of angular momentum in red giants}

Similarly to subgiant stars, the internal transport of angular momentum can also be investigated in red giant stars thanks to measurements of rotational splittings of mixed oscillation modes. This has first been done for the red giant KIC 8366239 by Beck et al. (2012). By comparing these asteroseismic data to rotational splittings computed for rotating models of KIC 8366239, one finds that an efficient 
angular momentum transport mechanism is also needed during the red-giant phase in addition to hydrodynamic transport processes (Eggenberger et al. 2012; Marques et al. 2013). While KIC 8366239 is a $1.5 \mathrm{M}_{\odot}$ star that is characterized by having a convective core during its evolution on the main sequence, the same conclusion is reached for the low-mass (about $0.84 \mathrm{M}_{\odot}$ ) red giant KIC 7341231 observed by Deheuvels et al. (2012) that has a radiative core on the main sequence (Ceillier et al. 2012, 2013).

As for subgiant stars, quantitative constraints can be put on the efficiency of the additional angular momentum transport process for red giants without being impacted by the past rotational evolution of the stars (Eggenberger et al. 2012, 2017). One then finds that, similarly to subgiant stars, the efficiency of the additional transport process increases with the mass of red giants. However, contrary to the trend found for subgiants, the efficiency of the angular momentum transport is found to increase when the star ascends the red-giant branch. This different behaviour determined for subgiant and redgiant stars may point towards a difference in the physical nature of the missing transport process during the subgiant and the red giant phase.

Concerning angular momentum transport by magnetic fields, it is interesting to note that, contrary to the solar case (see Sect. 1.3), the Tayler-Spruit dynamo leads to an additional angular momentum transport that is not sufficient to correctly account for the low core rotation rates deduced from asteroseismic data for a large sample of red giants by Mosser et al. (2012) and Gehan et al. (2018). This is shown for red-giant models of $1.5 \mathrm{M}_{\odot}$ (Cantiello et al. 2014) as well as for more massive $2.5 \mathrm{M}_{\odot}$ models of red giants (den Hartogh et al. 2019).

\section{Conclusion}

In this presentation, the constraints on the internal rotation that can be deduced from measurements of rotational splittings of solar-like oscillations have been briefly discussed in the context of magnetic angular momentum transport in stellar interiors. These measurements indicate that an efficient additional (in addition to the transport by meridional circulation and shear instability) process for angular momentum transport is needed for stars at different evolutionary stages. While angular momentum transport by magnetic instabilities constitutes an interesting candidate for the Sun and solar-type stars, the same process is not able to correctly reproduce the asteroseismic constraints on the internal rotation of subgiant and red giant stars. This illustrates the difficulty of modelling angular momentum transport in stellar radiative interiors and the need to develop the theoretical description of this transport together with the characterization of its efficiency thanks to asteroseismic measurements.

\section{Acknowledgements}

PE thanks the organizers for their kind invitation to give this talk and for the beautiful conference in Liège.

\section{References}

Acevedo-Arreguin, L. A., Garaud, P., \& Wood, T. S. 2013, MNRAS , 434, 720

Appourchaux, T., Belkacem, K., Broomhall, A.-M., et al. 2010, A\&A Rev., 18, 197

Appourchaux, T. \& Corbard, T. 2019, arXiv e-prints 
Beck, P. G., Montalban, J., Kallinger, T., et al. 2012, Nature, 481, 55

Belkacem, K. 2011, in Lecture Notes in Physics, Berlin Springer Verlag, Vol. 832, Lecture Notes in Physics, Berlin Springer Verlag, ed. J.-P. Rozelot \& C. Neiner, 139

Belkacem, K., Samadi, R., Goupil, M. J., et al. 2009, A\&A, 494, 191

Benomar, O., Takata, M., Shibahashi, H., Ceillier, T., \& García, R. A. 2015, MNRAS , 452, 2654

Braithwaite, J. 2006, A\&A, 449, 451

Braithwaite, J. \& Spruit, H. C. 2017, Royal Society Open Science, 4, 160271

Brown, T. M., Christensen-Dalsgaard, J., Dziembowski, W. A., et al. 1989, ApJ , 343, 526

Brun, A. S. \& Zahn, J.-P. 2006, A\&A, 457, 665

Cantiello, M., Mankovich, C., Bildsten, L., Christensen-Dalsgaard, J., \& Paxton, B. 2014, ApJ , 788, 93

Ceillier, T., Eggenberger, P., García, R. A., \& Mathis, S. 2012, Astronomische Nachrichten, 333, 971

Ceillier, T., Eggenberger, P., García, R. A., \& Mathis, S. 2013, A\&A, 555, A54

Charbonneau, P. \& MacGregor, K. B. 1993, ApJ , 417, 762

Couvidat, S., García, R. A., Turck-Chièze, S., et al. 2003, ApJ , 597, L77

Deheuvels, S., Doğan, G., Goupil, M. J., et al. 2014, A\&A, 564, A27

Deheuvels, S., García, R. A., Chaplin, W. J., et al. 2012, ApJ , 756, 19

den Hartogh, J. W., Eggenberger, P., \& Hirschi, R. 2019, A\&A, 622, A187

Eggenberger, P., Buldgen, G., \& Salmon, S. J. A. J. 2019a, A\&A, 626, L1

Eggenberger, P., Deheuvels, S., Miglio, A., et al. 2019b, A\&A, 621, A66

Eggenberger, P., Lagarde, N., Miglio, A., et al. 2017, A\&A, 599, A18

Eggenberger, P., Maeder, A., \& Meynet, G. 2005, A\&A, 440, L9

Eggenberger, P., Meynet, G., Maeder, A., et al. 2010, A\&A, 519, A116

Eggenberger, P., Montalbán, J., \& Miglio, A. 2012, A\&A, 544, L4

Elsworth, Y., Howe, R., Isaak, G. R., et al. 1995, Nature, 376, 669

Fossat, E., Boumier, P., Corbard, T., et al. 2017, A\&A, 604, A40

García, R. A., Salabert, D., Ballot, J., et al. 2011, GONG-SoHO 24: A New Era of Seismology of the Sun and Solar-Like Stars, 271, 012046

García, R. A., Turck-Chièze, S., Jiménez-Reyes, S. J., et al. 2007, Science, 316, 1591

Gehan, C., Mosser, B., Michel, E., Samadi, R., \& Kallinger, T. 2018, ArXiv e-prints 
Gough, D. O. \& McIntyre, M. E. 1998, Nature, 394, 755

Kosovichev, A. G. 1988, Soviet Astronomy Letters, 14, 145

Kosovichev, A. G., Schou, J., Scherrer, P. H., et al. 1997, Sol. Phys., 170, 43

Lund, M. N., Miesch, M. S., \& Christensen-Dalsgaard, J. 2014, ApJ , 790, 121

Maeder, A. \& Meynet, G. 2005, A\&A, 440, 1041

Marques, J. P., Goupil, M. J., Lebreton, Y., et al. 2013, A\&A, 549, A74

Mestel, L. 1953, MNRAS , 113, 716

Mestel, L. \& Weiss, N. O. 1987, MNRAS, 226, 123

Mosser, B., Goupil, M. J., Belkacem, K., et al. 2012, A\&A, 548, A10

Nielsen, M. B., Schunker, H., Gizon, L., \& Ball, W. H. 2015, A\&A, 582, A10

Rüdiger, G. \& Kitchatinov, L. L. 1996, ApJ , 466, 1078

Schunker, H., Schou, J., \& Ball, W. H. 2016, A\&A, 586, A24

Schunker, H., Schou, J., Gaulme, P., \& Gizon, L. 2018, Sol. Phys., 293, 95

Spada, F., Lanzafame, A. C., \& Lanza, A. F. 2010, MNRAS , 404, 641

Spruit, H. C. 1999, A\&A, 349, 189

Spruit, H. C. 2002, A\&A, 381, 923

Strugarek, A., Brun, A. S., \& Zahn, J.-P. 2011, A\&A, 532, A34

Tayler, R. J. 1973, MNRAS , 161, 365

Wood, T. S. \& Brummell, N. H. 2018, ApJ , 853, 97

Zahn, J., Brun, A. S., \& Mathis, S. 2007, A\&A, 474, 145

Zahn, J.-P. 1992, A\&A, 265, 115 